\title{
Experience with tracheostomy in medical intensive care patients
}

\author{
R.H. Gunawardana
}

\section{Department of Anaesthesiology, Faculty of Medicine, University of Peradeniya, Peradeniya, Sri Lanka}

\begin{abstract}
Summary: Thirty-four intensive care patients who received elective tracheostomies were studied to determine the incidence and nature of complications associated with tracheostomies. Indication for tracheostomy was long-term ventilation. Adverse consequences occurred in $47 \%$ of the patients. The most frequent problem was pneumonia. There were no intraoperative complications. Two deaths occurred due to delayed haemorrhage. Some complications of tracheostomy were influenced by the preceding period of endotracheal intubation. In patients requiring prolonged mechanical ventilation, tracheostomy carries a considerable risk. Optimal airway care only will reduce the incidence of complications.
\end{abstract}

\section{Introduction}

The use of endotracheal intubation or tracheostomy for respiratory support of critically ill patients is a life-saving form of therapy. However, both endotracheal intubation and tracheostomy cause frequent and severe complications, despite use of soft cuff tubes and meticulous respiratory care. $^{1-3}$ The complications and consequences of tracheostomy were found to be more severe than those of prolonged endotracheal tube intubation. ${ }^{3,4}$ There are reports of endotracheal intubation for more than 50 days with only minor sequelae, ${ }^{5}$ although some recommend early tracheostomy for patients requiring prolonged ventilation. ${ }^{6}$ Some studies have indicated that the complications from conversion to a tracheostomy are actually greater than those that could be expected from markedly increasing the length of endotracheal placement alone. ${ }^{3,7}$ Thus the choice of an airway in patients requiring mechanical ventilatory support and the optimal time to perform a tracheostomy remains controversial. However, the tracheostomy offers the advantages of lower airway resistance, reduction of dead space, less movement of the tube within the trachea, greater patient comfort, the ability to provide oral feedings and more efficient suctioning.

The present study was undertaken to determine the nature and incidence of complications associated with current tracheostomy. Only the early complications were studied while the patients were in the hospital.

Correspondence: R.H. Gunawardana, F.F.A.R.C.S. (England)

Accepted: 23 September 1991

\section{Method}

Over a five-year period, 34 critically ill patients with varying underlying problems (polyneuropathy 18 , organophosphate poisoning 15 , poliomyelitis 1) received tracheostomies: $73 \%$ were males; ages ranged from 3 to 70 years (mean 37). The indication for tracheostomy was for prolonged ventilation Three patients were in need of better pulmonary? toilet in addition to extended ventilatory support. The duration of endotracheal intubation prior to tracheostomy ranged from 12 to 24 days (mean 18).

All patients underwent tracheostomy in the operating theatre with the endotracheal tube in situ: $60 \%$ oral, $40 \%$ nasal. The anaesthetist provided pain relief, sedation and muscle relaxation as required. The trachea was opened by a vertical midline incision made through the second and third or the third and fourth rings with no removal of cartilage. A low-pressure cuff portex tube was inserted and the flanges were sutured to the skin. The cuff was adjusted to minimal leak in patients receiving mechanical ventilation. The intracuff pressure was not monitored. The ventilator tubings were supported to avoid transmission of gravitational forces to the tracheostomy tube.

An attempt was made to provide optimal postoperative care. Adequate humidification was provided. All efforts were made to aspirate secretions under sterile conditions. The tracheostomy wound dressings were carried out as often as necessary to keep the stoma dry. There was no fixed schedule for changing the tubes. All patients on mechanical ventilators received nutrition via nasogastric tube. No patient received cimetidine, antacids or prophylactic anticoagulant therapy.

All patients required mechanical ventilation. 
The duration of mechanical ventilation following tracheostomy ranged from 8 to 50 days (mean 21). The cuff was deflated when mechanical ventilation was no longer required. The duration of tracheostomy was 16 to 62 days (mean 28). Twelve patients required more than 30 days of tracheostomy management. Extubation was by simple removal of the tube. No weaning protocol with fenestrated tubes or tracheostomy buttons was employed.

\section{Results}

A total of 24 complications occurred in 16 patients. There were no intraoperative complications such as haemorrhage, pneumothorax, pneumomediastinum, subcutaneous emphysema and respiratory arrest. Three patients under the age of 10 years developed six complications consisting of obstruction of the tube with mucous plugs, atelectasis and respiratory distress.

Pneumonia was the most common complication observed in this series. Nine patients with tracheostomies developed pneumonia, which was observed more frequently with increasing duration of ventilation. The diagnosis of pneumonia was made on the basis of fever, leucocytosis, purulent sputum, the physical and radiological signs of pulmonary infection and the presence of pathogenic organisms in the tracheal aspirate cultures. The organisms isolated were Staphylococcus aureus, Escherichia coli and Pseudomonas aeruginosa. Four patients had pneumonia at the time of tracheostomy, three of whom had signs suggestive of aspiration on admission. Increased tracheal secretions were frequently found in patients following organophosphate poisoning. Three patients developed stomal infection with purulent discharge. All patients received systemic antibiotic therapy on the basis of cultures.

Two patients with marked pulmonary and stomal sepsis developed sudden massive fatal arterial haemorrhage during the second postoperative week. Both patients were receiving mechanical ventilation for respiratory failure following organophosphate poisoning. Several litres of red blood were lost during a $10-15$ min period. There were no warning signs such as minor tracheal bleeding or pulsation of the tracheostomy tube and they had no recognized bleeding tendency. Hyperinflation of the tracheostomy cuff, finger compression of the innominate artery and blood replacement were carried out while preparations were being made for surgical repair. Both died of aspiration and exanguinating haemorrhage before corrective action could be taken. Autopsy revealed necrosis of the tracheal wall at the sites of the tip and cuff and erosion of the innominate artery in addition to pneumonia.

In the survivors, the tracheostomy wound was firmly healed three weeks after extubation. None of them had clinically detectable tracheal complications.

\section{Discussion}

Longer intubation periods appear to be the trend, and several studies indicate that lower morbidity and mortality are achieved through intubation rather than through early tracheostomy. ${ }^{3,8}$ However, tracheostomy is superior to orotracheal intubation with regard to patient comfort, ease of swallowing, management of oral secretions and ease of suctioning and mouth care. Also, a 25\% incidence of paranasal sinusitis is reported in neurosurgical patients who were nasotracheally intubated. ${ }^{9}$ The reported incidence of tracheostomy complications varies from $6 \%{ }^{10}$ to $51 \%{ }^{4}$ The complications of tracheostomy may have been influenced by the preceding period of endotracheal intubation, since tracheostomy was frequently performed after a period of endotracheal intubation. Pre-existing endotracheal damage to the airway was not evaluated prior to surgery. In the present study no standard policy regarding timing of tracheostomy was employed, the decision to perform tracheostomy was made after considering all factors relevant to each patient.

No peroperative complications were encountered in the present series because all tracheostomies were done as elective procedures under optimal conditions with a controlled airway. The reported rate of complications with emergency tracheostomy is two to five times as high as with elective operation. ${ }^{11}$ Tracheostomy in the paediatric patient has been associated with significant morbidity and mortality compared to that in adults. In keeping with other series, ${ }^{12-14}$ the incidence of complications in children was higher but there were no tracheostomy related deaths.

Inadvertent tube displacement, a major early complication of tracheostomy, was not encountered in the present series. Suturing of the flanges to the skin prevents displacement of the tube. ${ }^{15}$ Displacement during the first 5 days after surgery is hazardous because the tracheostomy wound has not yet developed a secure tract. Efforts to reinsert a tracheostomy tube generate a false channel anterior to the trachea thereby compressing the trachea.

The incidence of pneumonia in the present study is high. A major factor leading to pneumonia is the duration of mechanical ventilation. The incidence of pneumonia is higher in patients with tracheostomies because they are ventilated for longer than patients with endotracheal intubation. ${ }^{16}$ The 
risk of nosocomial pneumonia was found to be $66 \%$ in patients with tracheostomy and a respirator, with a significantly higher incidence after the fifth day of therapy, while no case of nosocomial pneumonia occurred in patients on respirator for less than $24 \mathrm{~h} .{ }^{17}$ When a patient is placed on a ventilator all defence mechanisms are circumvented and the alveoli are exposed directly to the outside environment. The prolonged use of mechanical ventilation results in infection of the lungs and tracheobronchial tree.

Serious disease predisposes to the rapid acquisition of Gram-negative bacilli. Colonization of the respiratory tract with pathogens is common in acutely ill patients on ventilators. Critically ill patients with tracheostomies develop more serious pneumonia with enteric Gram-negative bacilli, especially Pseudomonas species. ${ }^{18}$ In addition, increased adherence of Gram-negative bacilli to the tracheal mucosa is seen in tracheostomized patients ${ }^{19}$ and the degree of binding seems to be related to the patient's nutritional status. ${ }^{20}$ Tracheostomy is associated with very early depression of the tracheal mucociliary clearance. ${ }^{21}$ The natural filtering mechanism too is bypassed with the introduction of the tube. Mechanical ventilators, nebulisers and endotracheal suctioning have been suggested as frequent sources of tracheal contamination. However, even with sterile equipment, colonization and infection continue to occur in patients receiving mechanical ventilation. Therefore, aseptic techniques in tracheostomy care are important to minimize pulmonary infection in patients on mechanical ventilators. Furthermore, aspiration too plays a role in the pathogenesis of infection. Prolonged intubation in the presence of infected pulmonary secretions has been noted to be undesirable and may help predispose to an increased incidence of complications. ${ }^{8,22}$

The reported incidence of delayed fatal haemorrhage is low. ${ }^{23}$ Delayed haemorrhage is most often due to erosion of a major vessel by pressure necrosis from the cuff or directly from the tip of the tube. The innominate artery is usually affected. Low tracheostomy and hyperextension of the neck at the time of the incision are factors that contribute towards this potentially life-threatening complication. The innominate artery crosses the anterolateral surface of the trachea at the level of the upper sternum and a tracheostomy tube placed too low can erode directly into the artery. Arterial bleeding was not a complication when tracheostomies were made at the second ring. ${ }^{6}$

Severe sepsis, prolonged cuff pressure and possible overinflation probably resulted in massive fatal arterial haemorrhage in two instances in the present study. Probably secondary infection of already traumatized and devitalized tracheal tissue already present at the time of conversion to a tracheostomy, further contributed to massive destruction.

Most of the tracheal damage occurs at the site of the cuff. The pressure exerted on the tracheal wall by the inflated cuff is generally recognized as the primary cause of the tracheal damage. ${ }^{24,25}$ The relation between high cuff to tracheal wall pressure and tracheal injury is well documented. ${ }^{3,26,27}$ During mechanical ventilation intracuff pressure must be low enough to allow tracheal capillary perfusion and high enough to prevent loss of tidal volume. Ischaemic damage will occur if cuff to tracheal wall pressure exceeds capillary perfusion pressure (20-30 $\mathrm{mmHg}$ ) for a significant time. ${ }^{27}$ The damage varies from superficial erosion to tracheal perforation resulting in complications such as erosion of blood vessel, tracheal stenosis and tracheoesophageal fistula. Careful inflation of the cuff to allow an audible leak during inspiration eliminates significant tracheal injury. The high-volume lowpressure portex cuffs also caused damage unless the cuff could occlude at pressures less than $25 \mathrm{mmHg}$ intracuff pressure. ${ }^{26}$ The cuff pressure should be monitored with each inflation and maintained below $25 \mathrm{mmHg} .^{26,28}$ Accidental overinflation of the cuff is a principal cause of tracheal injury in patients who require mechanical ventilation. Hourly cuff deflation failed to prevent mucosal necrosis and cartilage injury. ${ }^{26}$

When haemorrhage develops, median stenotomy should be performed with ligation and resection of affected artery ${ }^{29}$ Even with appropriate management only $25 \%$ of patients survive. ${ }^{29}$

Tracheostomy is not a totally benign procedure in the management of patients who required longterm ventilation. Close attention to surgical technique and long-term patient care can only reduce the number of complications.

4. Dane, T.E.B. \& King, E.G. A prospective study of complications after tracheostomy for assisted ventilation. Chest 1975 , 67: 398-404.

5. Via-Ruque, E. Prolonged oro or nasotracheal intubation. Crit Care Med 1981, 9: 637-639.

6. Dayal, V.S. \& El Masiri, W. Tracheostomy in intensive care setting. Laryngoscope 1986, 96: 58-60.
Whited, R.E. A prospective study of laryngotracheal sequela in long-term intubation. Laryngoscope 1984, 94: 367-377. prolonged intubation. Laryngoscope 1985, 95: 1461-1467. . Stauffer, J.L., Olson, D.E. \& Petty, T.K. Complications and consequences of endotracheal intubation and tracheotomy. A prospective study of 150 critically ill adult patients. Am J Med 1981, 70: 65-76. 
7. Andrews. M.J. \& Pearson, F.G. Incidence and pathogenesis of tracheal injury following cuffed tube tracheostomy with assisted ventilation. Ann Surg 1971, 173: 249-251.

8. El-Naggar, M., Sadagopan, S., Levine, H., Kantor, H. \& Collins, V.J. Factors influencing choice between tracheostomy and prolonged translaryngeal intubation in acute respiratory failure. Anaesth Anal 1976, 55: 195-201.

9. Deutschmann, C.S., Wilton, P.B., Sinow, J., Thienprasit, P., Konstantinides, F.N. \& Cerra, F.B. Paranasal sinusitis: a common complication of nasotracheal intubation in neurosurgical patients. Neurosurgery 1985, 17: 296-299.

10. Yarington, C.T. \& Frazer, J.P. Complications of tracheostomy. Arch Surg 1965, 91: 652-654.

11. Skaggs, J.A. Tracheostomy: management, mortality and complications. Am Surg 1969, 35: 393-396.

12. Glimore, B.B. \& Mickelson, S.A. Pediatric tracheostomy. Controversies in management. Otolaryngol Clin North Am 1986, 19: $141-151$.

13. Chew, J.Y. \& Cantrell, R.W. Tracheostomy: complications and their management. Arch Otolaryngol 1972, 96: 538-545.

14. Orlowski, J.P., Ellis, N.G., Amin, N.P. \& Crumrine, R.S. Complications of airway intrusion in 100 consecutive cases in a pediatric ICU. Crit Care Med 1980, 8: 324-331.

15. Orringer, M.B. Endotracheal intubation and tracheostomy. Indications, techniques and complications. Surg Clin North Am 1980, 60: 1447-1464.

16. Zwillich, C.W., Pierson, D.J., Creagh, C.E., Sutton, F.D., Schatz, E. \& Petty, T.L. Complications of assisted ventilation. A prospective study of 354 consecutive episodes. Am J Med 1984, 57: $161-170$.

17. Cross, A.S. \& Roup, B. Role of respiratory assistance devices in endemic nosocomial pneumonia. Am J Med 1981, 70: 681-685.

18. Neiderman, M.S., Ferranti, R.D., Zeigler, A., Merrill, W.W. \& Reynolds, H.Y. Respiratory infection complicating longterm tracheostomy. The implication of persistent Gram negative tracheobronchial colonisation. Chest 1984, 85: $39-44$.
19. Niederman, M.S., Rafferty, T.D., Sasaki, C.T., Merrill, W.W., Matthay, R.A. \& Reynolds, H.Y. Comparison of bacterial adherence to ciliated and squamous epithelial cells obtained from the human respiratory tract. Am Rev Respir Dis 1983, 127: 85-90.

20. Niederman, M.S., Merrill, W.W., Ferranti, R.D., Pagano, K.M., Palmer, L.B. \& Reynolds, H.R. Nutritional status and bacterial binding in the lower respiratory tract in patients with chronic tracheostomy. Ann Intern Med 1984, 100: 795-800.

21. Alexopoulos, C., Jansson, B. \& Lindholm, C.E. Mucus transport and surface damage after endotracheal intubation and tracheostomy. An experimental study in pigs. Acta Anaesthesiol Scand 1984, 28: 68-76.

22. Sasaki, C.T., Horiuchi, M. \& Koss, N. Tracheostomy related subglottic stenosis: bacteriologic pathogenesis. Laryngoscope 1979, 89: 857-865.

23. Mathog, R.H. Delayed massive haemorrhage following tracheostomy. Laryngoscope 1971, 81: 107-119.

24. Carroll, R., Hedden, M. \& Safar, P. Intratracheal cuffs: performance and characteristics. Anaesthesiology 1969, 31: 275-277.

25. Cooper, J.D. \& Grillo, H.C. The evolution of tracheal injury due to ventilatory assistances through cuffed tubes. Ann Surg 1969, 169: 334-336.

26. Ching, N.P.H., Ayres, S.M., Spina, R.C. \& Nealon, T.F. Endotracheal damage during continuous ventilatory support. Ann Surg 1974, 179: 123-127.

27. Nordin, U. The trachea and cuff induced tracheal injury. Acta Otolaryngol Stockh 1977, (Suppl 345): 7-56.

28. Bernhard, W.N., Yost, L., Joynes, D., Cothalis, S. \& Turndof, $H$. Intracuff pressures in endotracheal and tracheostomy tubes: related cuff physical characteristics. Chest 1985, 87: 720-730.

29. Jones, J.W., Reynolds, M., Hewwitt, R.L. \& Drapans, T. Tracheo-innominate artery erosion: successful surgical management of a devastating complication. Ann Surg 1977, 184: $194-204$. 